\title{
Use of Dapsone for Difficult to Treat Stricturing Esophageal Crohn's Disease: A Difficult Pill to Swallow
}

\author{
Eran Zittan ${ }^{*}$ and Mark S Silverberg \\ Division of Gastroenterology, Mount Sinai Hospital, University of Toronto, Canada
}

*Corresponding author: Eran Zittan, Division of Gastroenterology, Mount Sinai Hospital, University of Toronto, 441-600 University Avenue, Toronto, Ontario, M5G 1X5, Canada, Tel: 416-586-4800, Fax: 416-619-5524

\begin{abstract}
Crohn's disease (CD) can affect any part of the gastrointestinal tract. Although esophageal involvement is rare, it can lead to debilitating complications such as tracheobronchial fistulation. Although current guidelines recommend early, advanced therapy with acid suppression, immunosuppressants and biologics, there are a lack of specific clinical trial data demonstrating efficacy for this phenotype. Despite aggressive management, CD of the esophagus is often refractory to conventional therapy. We report a case of stricturing esophageal Crohn's disease refractory to conventional therapy that was successfully treated with endoscopic dilatation and oral dapsone.
\end{abstract}

\section{Case}

A 25-year-old woman diagnosed with Crohn's disease in 2000 presented with weight loss, nausea, vomiting, diarrhea, oral ulcers, and sitophobia. At the time of initial endoscopy she was found to have disease affecting her colon with deep ulcers extending from the sigmoid colon to the hepatic flexure as well as ulcers in her terminal ileum. She also had esophageal ulcers but no luminal narrowing ( $\mathrm{L} 3 / \mathrm{L} 4$ disease as per the Montreal Classification). She was initially started on oral prednisone but there was no improvement and she developed mania and suicidal ideation consistent with steroid psychosis. She also had no response to budesonide or acid suppression. From 2001 to 2003 she was on infliximab and methotrexate. Infliximab was discontinued because of anti-histone positive drug induced lupus. She was switched to adalimumab from 2006 to 2008 but this had to be stopped due to ongoing arthritis. From 2008 to 2010 she was treated with ustekinumab and metho- trexate but continued to have evidence of both clinical and endoscopically active disease. In 2010 she received a temporary diversion ileostomy and in 2012 she went on to have a total proctocolectomy with end-ileostomy due to clinical improvement with the temporary stoma. Following the surgery she was maintained in clinical remission on topical fluocinonide $0.05 \%$ gel, azathioprine $100 \mathrm{mg}$ daily, golimumab $200 \mathrm{mg}$ every two weeks and esomeprazole $40 \mathrm{mg}$ daily. She returned in 2013 with new onset dysphagia, significant weight loss and diffuse arthritis. Prior to this she had no esophageal symptoms. EGD was performed in August 2013 which showed severe ulceration and stricturing of the mid-esophagus requiring dilatation using a 30,32 and $34 \mathrm{~mm}$ Bougie dilators (Figure $1 \mathrm{~A}$, Figure $1 \mathrm{~B}$ and Figure $1 \mathrm{C}$ ). She had repeat dilatation 1 month later. Biopsies from these procedures revealed acute and active chronic inflammatory infiltrate with no granulomas, inclusion bodies or evidence of malignancy. Given the isolated nature of the pathology in the mid-esophagus while on acid suppression therapy and lack of features consistent with other conditions, the esophagitis and stricture was thought to be consistent with foregut CD. At this time golimumab levels were measured using an assay designed for infliximab measurements and the result was $15.6 \mu \mathrm{g} / \mathrm{ml}$ (Prometheus Laboratories).

The patient had repeat EGD in October 2013 which showed no improvement. Ileostomy in January 2014 was normal (Figure 2). Blood work at this time revealed white cells $7.99 \times 10^{9} / \mathrm{L}$, platelets $419 \times 10^{9} / \mathrm{L}$, hemoglobin 11.3 $\mathrm{g} / \mathrm{dL}, \mathrm{MCV} 81 \mathrm{fL}$, CRP $0.3 \mathrm{mg} / \mathrm{L}$, normal electrolytes and liver enzymes. After ruling out G6PD deficiency, dapsone 
A

\section{B}
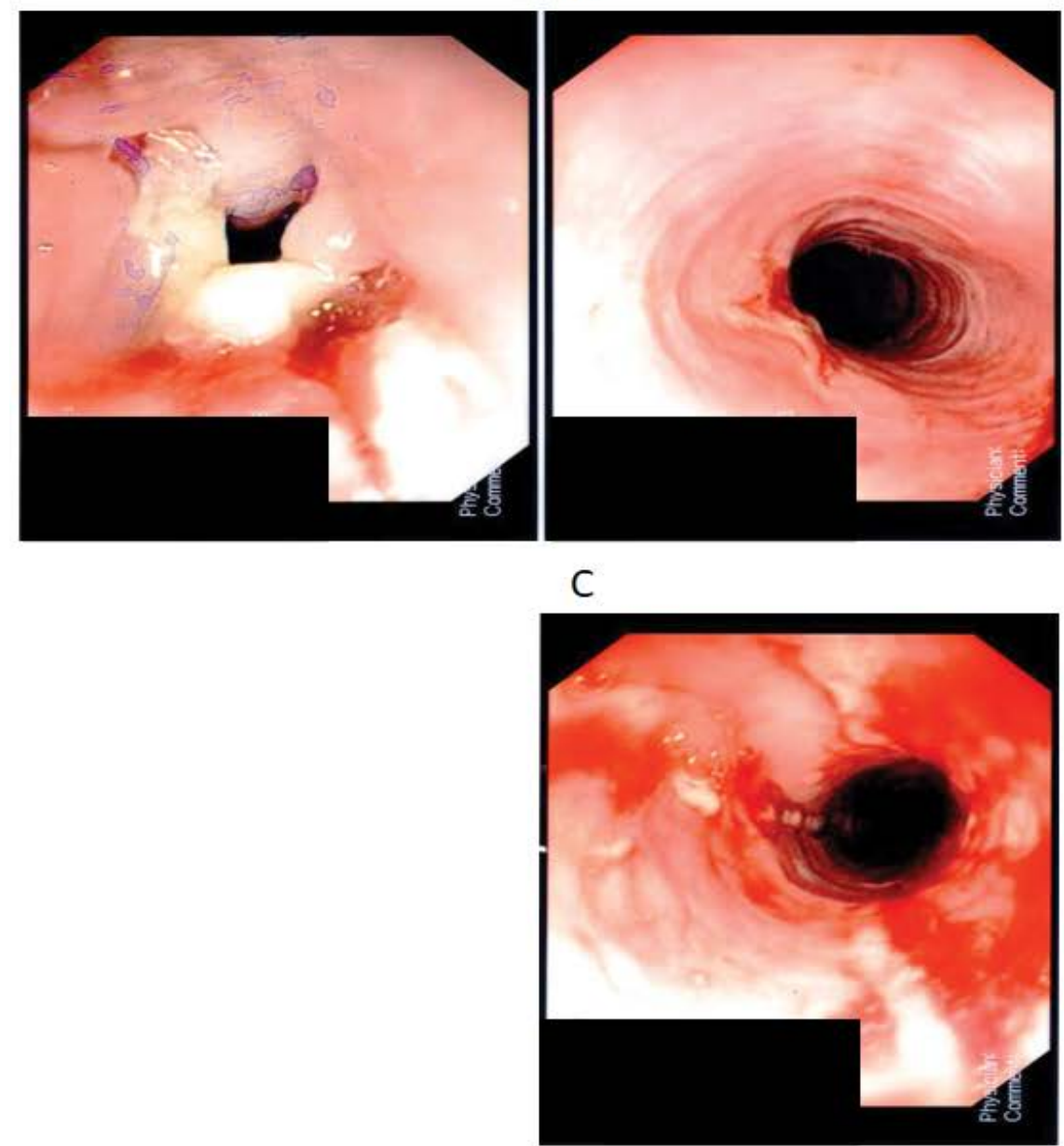

Figure 1: A) Esophageal ulceration and stricture pre-dilatation; B) Esophagus distal to the stricture; C) Esophageal stricture post-dilatation.
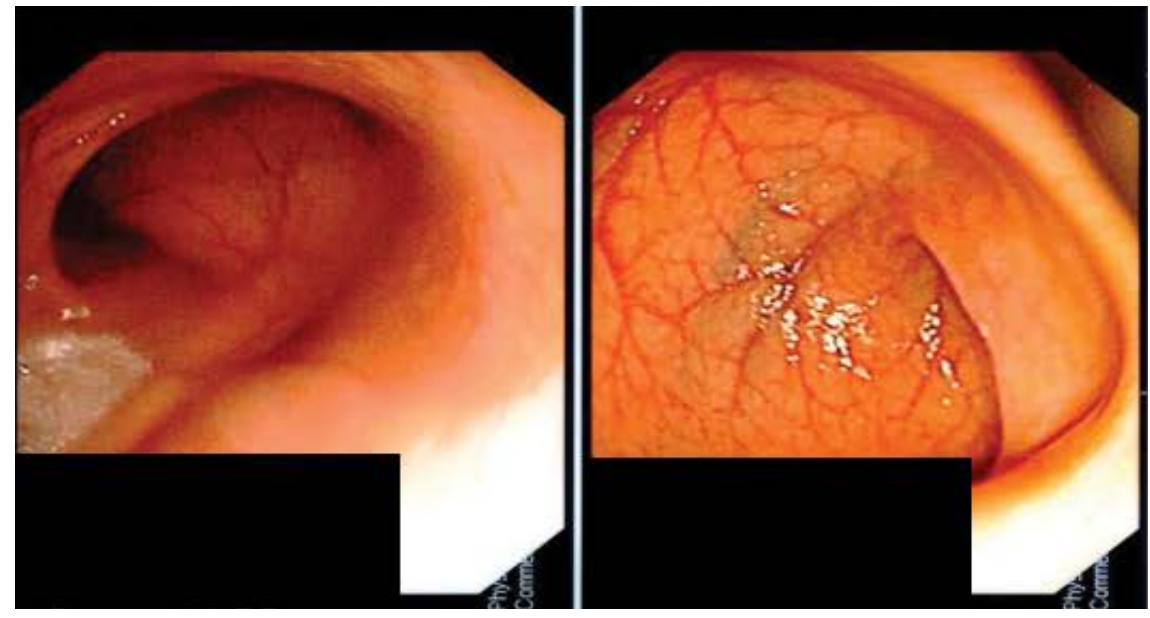

Figure 2: Normal ileoscopy.

$25 \mathrm{mg}$ PO daily was initiated. The dose was increased by $25 \mathrm{mg}$ every 4 days until a maximum dose of $100 \mathrm{mg}$ daily. During this time blood work was monitored weekly for complete blood count (CBC), electrolytes, and liver enzymes. After 3 months, the interval was increased to every 3 months. In January 2014, the dose of dapsone was decreased to $75 \mathrm{mg}$ daily because of difficulty swallowing large pills. Her esophageal symptoms slowly improved over several months and she had significant weight gain.
A repeat EGD and ileostomy was performed in December 2014. The ileostomy remained normal whereas the EGD showed only mild patchy inflammation in the esophagus with no evidence of stricture (Figure 3). In February 2014, golimumab was discontinued because of recurrent anti-histone antibody positive inflammatory arthritis. Currently as of July 2015 she is asymptomatic and is maintaining a healthy weight on esomeprazole $40 \mathrm{mg}$ daily, azathioprine $100 \mathrm{mg}$ daily, and dapsone $100 \mathrm{mg}$ daily. 

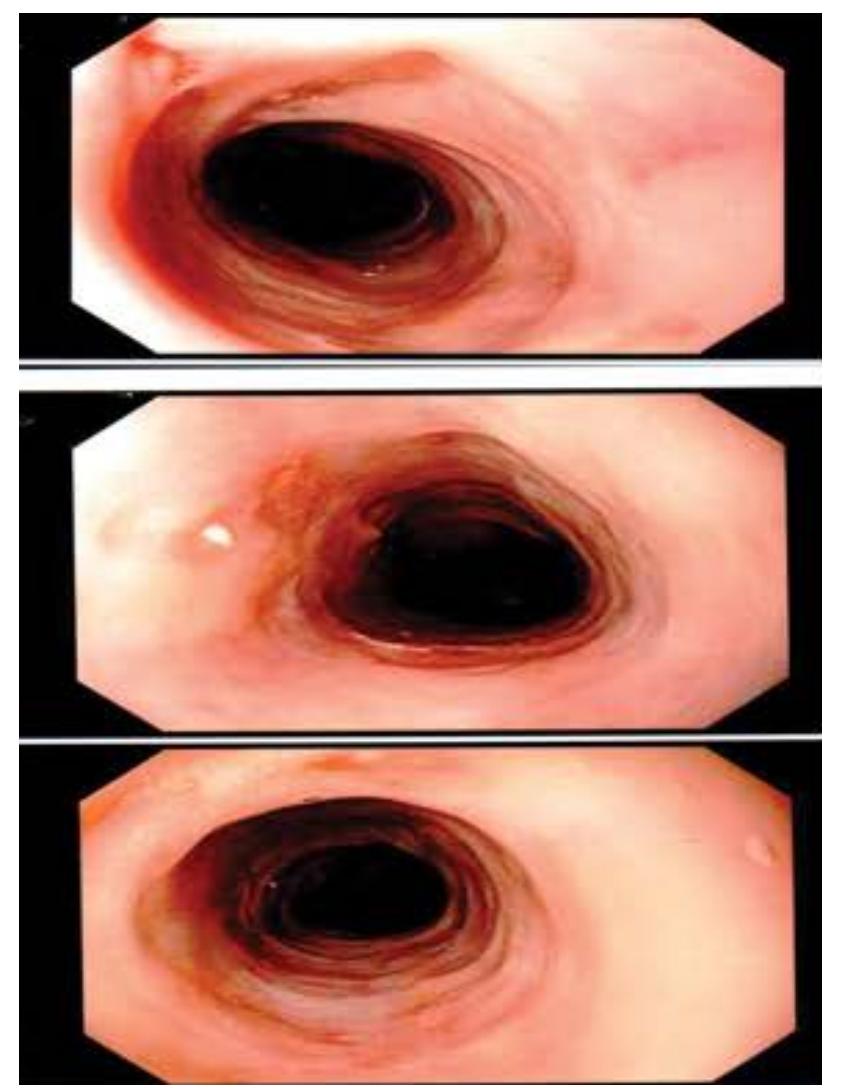

Figure 3: Esophagus post-treatment with Dapsone showing healing of ulcer and absence of stricture.

\section{Discussion}

Esophageal involvement in CD affects up to $2 \%$ of the total patient population and is associated with poor prognosis $[1,2]$. These patients can be asymptomatic or experience debilitating dysphagia, odynophagia and reflux [3]. Patients with foregut involvement almost invariably have distal disease as well. Current guidelines do not recommend EGD as part of the evaluation to assess extent of disease in adults, however, it is worth considering in patients with features of IBD unclassified, dyspepsia or other esophageal symptoms [4]. Based on case series, the most common histologic findings are active chronic inflammation and ulcerations whereas non-caseating granulomas and intraepithelial lymphocytes are seldom present $[5,6]$. Rarely esophageal CD can fistulize into surrounding structures such as the tracheobronchial tree. If this is suspected, further imaging studies such as CT or MRI should be performed [4]. Patients with moderate to severe disease should be treated aggressively to manage symptoms and avoid such complications. These patients should be treated with acid suppression and early immunosuppression with a thiopurine or methotrexate and early consideration of biologic therapy. Treating physicians should have a low threshold for using anti-TNF therapy given the worse prognosis [4]. Only about half of patients with esophageal $C D$ respond to conventional therapy and therefore an alternative approach should be considered [6]. Case reports have described successful treatment of refractory esophageal CD using thalidomide and granulocyte/monocyte absorption $[7,8]$. These novel therapies have shown utility in treating typical intestinal CD owing to their shared immunomodulatory and anti-inflammatory properties [9-13]. Thalidomide is thought to act mainly through decreasing production of TNF alpha and augmenting T cell response $[9,10]$, whereas granulocyte/monocyte absorption actively removes these cells from the circulation as these cells are thought to have an integral role in mucosal inflammation in $C D$ [11]. Dapsone (4,4'-diaminodiphenylsulfone) is an antibiotic typically used for the treatment of leprosy. It has also been widely used to treat several non-infectious, dermatologic conditions owing to its anti-inflammatory properties [12]. Numerous studies exist that describe a multitude of possible mechanisms of action by which dapsone exerts an anti-inflammatory effect. These include but are not limited to its effects on immune effector cells, adhesion molecules/chemotaxis and inflammatory mediators such as reactive oxygen species and cytokines, in particular TNF alpha $[13,14]$. Early studies using dapsone showed promise in treating luminal and cutaneous manifestation of CD [15]. For treatment of leprosy, dapsone is dosed at $100 \mathrm{mg}$ per day, whereas in dermatitis herpetiformis, it is suggested to start at $50 \mathrm{mg}$ per day and titrate up to $300 \mathrm{mg}$ per day to achieve the desired effect. As with most medications, careful consideration prior to the initiation of dapsone should be used given the variety of potential adverse effects. G6PD deficiency should be ruled out given the increased risk for severe hemolytic anemia. Hypersensitivity and peripheral neuropathy may also occur and therefore patients should be monitored closely. Labs to measure CBC, liver and renal function should be obtained at baseline and monitored regularly (every 3-6 months) during therapy provided the dose is not increased. The CBC should be monitored more closely during the first few weeks after initiation of therapy and dapsone should be discontinued if there is evidence of hematologic dyscrasia. To our knowledge, this is the first case report showing the successful use of dapsone in the treatment of esophageal CD. Our refractory Crohn's disease patient that was presented with a very complicated esophageal CD was a primary non-respond to anti TNF such as Adalimumab, Infliximab, and Golimumab. Dapsone therapy significantly improved her esophageal ulcer, stricture and her inflammatory biomarker.

Given the morbidity, potential for severe complications and refractory nature to usual medication, dapsone may prove to be a potentially beneficial therapy in the treatment of esophageal CD. In addition, by elucidating the mechanism by which dapsone works to improve esophageal inflammation, further insight may be gained regarding the pathogenesis of $C D$.

\section{Acknowledgment}

The authors thank the patient for her permission to publish this case report and thank Dr. William Sandborn (UCSD) for his suggestion to try dapsone in this case. 


\section{References}

1. Jess $\mathrm{T}$, Winther $\mathrm{KV}$, Munkholm $\mathrm{P}$, Langholz $\mathrm{E}$, Binder $\mathrm{V}$ (2002) Mortality and causes of death in Crohn's disease: Follow-up of a population-based cohort in Copenhagen County, Denmark. Gastroenterology 122: 1808-1814.

2. Lou GC, Yang JM, Huang W, Zhang J, Zhou B (2009) Esophageal Crohn's disease. Endoscopy 41: E257.

3. Souza JL, da Silva JG, Sipahi AM (2009) Crohn's disease of the esophagus without inflammatory activity confirmed by the use of endoscopy with narrow-band imaging. Endoscopy 41: E188.

4. Van Assche G, Dignass A, Reinisch W, van der Woude CJ, Sturm A, et al. (2010) The second European evidence-based Consensus on the diagnosis and management of Crohn's disease: Special situations. J Crohns Colitis 4: 63-101.

5. De Felice KM, Katzka DA, Raffals LE (2015) Crohn's Disease of the Esophagus: Clinical features and treatment outcomes in the biologic era. Inflamm Bowel Dis 21: 2106-2113.

6. Decker GA, Loftus EV Jr, Pasha TM, Tremaine WJ, Sandborn WJ (2001) Crohn's disease of the esophagus: Clinical features and outcomes. Inflamm Bowel Dis 7: 113-119.

7. Barkin JA, Schonfeld WB, Deshpande AR (2013) Successful use of thalidomide for refractory esophageal Crohn's disease. Am J Gastroenterol 108: 855-857.

8. Moribata K, Kato J, limura S, Yoshida S, Shingaki N, et al. (2011) Mucosal healing of esophageal involvement of
Crohn's disease with granulocyte/monocyte adsorption. J Clin Apher 26: 225-227.

9. Meierhofer C, Wiedermann CJ (2003) New insights into the pharmacological and toxicological effects of thalidomide. Curr Opin Drug Discov Devel 6: 92-99.

10. Settles B, Stevenson A, Wilson K, Mack C, Ezell T, et al. (2001) Down-regulation of cell adhesion molecules LFA-1 and ICAM-1 after in vitro treatment with the anti-TNF-alpha agent thalidomide. Cell Mol Biol (Noisy-le-grand) 47: 1105-1114.

11. Fukuda $Y$, Matsui T, Suzuki $Y$, Kanke $K$, Matsumoto $T$, et al. (2004) Adsorptive granulocyte and monocyte apheresis for refractory Crohn's disease: An open multicenter prospective study. J Gastroenterol 39: 1158-1164.

12. Debol SM, Herron MJ, Nelson RD (1997) Anti-inflammatory action of dapsone: Inhibition of neutrophil adherence is associated with inhibition of chemoattractant-induced signal transduction. J Leukoc Biol 62: 827-836.

13. Abe M, Shimizu A, Yokoyama $Y$, Takeuchi $Y$, Ishikawa $O$ (2008) A possible inhibitory action of diaminodiphenyl sulfone on tumour necrosis factor-alpha production from activated mononuclear cells on cutaneous lupus erythematosus. Clin Exp Dermatol 33: 759-763.

14. Suda T, Suzuki Y, Matsui T, Inoue T, Niide O, et al. (2005) Dapsone suppresses human neutrophil superoxide production and elastase release in a calcium-dependent manner. $\mathrm{Br}$ J Dermatol 152: 887-895.

15. Prantera C, Argentieri R, Mangiarotti R, Levenstein S (1988) Dapsone and remission of Crohn's disease. Lancet: 536. 